\title{
Thermal Reshaping of Gold Nanorods in Micellar Solution of Water/Glycerol Mixtures
}

\author{
Al Sayed A. Al-Sherbini \\ Department of Environmental, Photochemical and Agriculture Applications, National Institute of Laser Enhanced Sciences (NILES), \\ Cairo University, P.O. Box 12631, Cairo, Egypt \\ Correspondence should be addressed to Al Sayed A. Al-Sherbini, elsayed@niles.edu.eg
}

Received 7 November 2009; Accepted 25 May 2010

Academic Editor: Renzhi Ma

Copyright ( $\odot 2010 \mathrm{Al}$ Sayed A. Al-Sherbini. This is an open access article distributed under the Creative Commons Attribution License, which permits unrestricted use, distribution, and reproduction in any medium, provided the original work is properly cited.

Gold nanorods (Nds) with aspect ratios of 4, 3.5, and 2.8 were prepared by the electrochemical method. The nanorods were thermally studied in binary solvents of aqueous glycerol at different ratios (25\%-75\%). The results illustrated that the longitudinal surface plasmon resonance $\left(\mathrm{SP}_{L}\right)$ is strongly dependent on the dielectric constant. The maximum absorption is red shifted with increasing the glycerol/water ratio. This was attributed to the decreasing value of the dielectric constant of the binary solvents. Moreover, by increasing the temperatures, the results showed relative instability of the gold nanorods. This attributed to the relative instability of the micelle capping the nanorods.

\section{Introduction}

Colloidal gold nanoparticles have received widespread attention in recent years due to both of their unusual properties and their promising applications [1-5]. Nanoparticles, typically in the size range of $1-100 \mathrm{~nm}$, possess size- and shape-dependent properties which differ markedly from their bulk behavior $[1,2,6-8]$. It is documented that goldnanoparticles give different intense absorption for both the plasmon transverss and longitudinal bands at different aspect ratios $[9,10]$. The total extinction cross section (which is the sum of the absorption and the scattering) is given as a summation over all electric and magnetic multiple oscillations. It is known that the optical response of spherical gold nanoparticles has red color with aspect ratio (length divided by the width) $=1$ and exhibits single absorption band at wavelength $\sim 520 \mathrm{~nm}$. This is attributed to the collective motion of the electrons in the conduction band. However, gold nanorods have different colors ranging from blue to red. The longitudinal band red shifted with increasing the aspect ratio of the nanorods. Mie $[11,12]$ first described this phenomenon theoretically by solving Maxwell's equation for a radiation field interacting with spherical metal particles under appropriate boundary conditions. The only materialrelated property and constants appearing in the Mie's theory are the complex dielectric function of metal and the dielectric constant of the surrounding medium. Maxwellgarnett theory $[10,13,14]$, an effective medium theory, is also often used to describe the optical properties of metallic nanoparticles. This theory computes the effective (complex) dielectric function of the composite material consisting of the metallic nanoparticles and the surrounding medium (host material). From this dielectric function, the refractive index and the absorption maxima can be calculated. Link and el Sayed [12] studied the effect of the medium dielectric constant on the absorption maximum of the longitudinal plasmon resonance and the results showed that longitudinal plasmon resonance depends on the medium dielectric constant in a linear fashion for a fixed aspect ratio. The study of micellar formation by anionic and cationic surfactants in aqueous binary solvents were carried out and the results obtained illustrated that the micelle formation in water is governed by the cohesive interactions between the hydrophobic tail of the monomers. Hence, the thermodynamics of micelle formation can be considered as the formation of a distinct micellar phase at critical 
micelles concentration (c.m.c), which is in equilibrium with monomers and the concentration of the monomers in binary mixture remains constant after micellisation. The formation and the dissociation of micelles depends on the two characteristic features of the organic solvents. The first feature is that the smaller the dielectric constant $(\varepsilon)$ of the organic solvent, the easier to break the micelles by lowering the overall $(\varepsilon)$ of the aqueous medium. The second feature is that an organic solvent with a large hydrophobic surface can easily break the micelles effectively [13].

The solvation of a binary solvent mixture has been found to be important in explaining spectroscopic, equilibrium, and kinetic data in solution chemistry. In a binary solvent, the solute can interact to a different degree with the two solvents of the binary mixture which has different dielectric constants and the solvation microsphere determines these interactions. The solute molecule will be preferentially solvated by the solvent with the strongest interaction depending on the polarity as well as the dielectric constant of the solvent [14].

In the present study, the effects of binary solvents of different dielectric constants on the absorption spectra of gold nanorods encapsulated in micelles were reported [15]. Rods with average aspect ratios of $4,3.5$, and 2.8 have been studied using glycerol water mixtures of different ratios and at different temperatures.

\section{Experimental}

The gold nanorods (Nds) have been prepared in micelles using the electrochemical method described previously [16]. The synthesis is conducted within a simple two-electrodestype electrochemical cell and consisted of a gold plate $3 \times 1 \times 0.05 \mathrm{~cm}$ (anode) and a platinum plate of the same dimensions (cathode). An additional silver plate of the same size is placed behind the platinum electrode. Both electrodes are immersed in the electrolytic solution to a depth of about $1.5 \mathrm{~cm}$ during the electrolysis. The electrolyte solution consisted of a hydrophilic cationic surfactant, hexadecyltrimethyl-ammonium bromide $\left(\mathrm{C}_{16} \mathrm{TAB}, 99 \%\right.$; Sigma), and a hydrophobic cationic surfactant, tetradecylammonium bromide ( $\mathrm{TC}_{10} \mathrm{AB},>98 \%$ fluka), or tetraoctylammonium bromide ( $\mathrm{TC}_{8} \mathrm{AB},>98 \%$ fluka) (as nanorods inducing surfactant). A few microliter, of acetone (puriss fluka) was added to increase the solubility of cylindrical shape-inducing cosurfactant into the $\mathrm{C}_{16} \mathrm{TAB}$ micellar framework and inducing the cylindrical growth to form the $\mathrm{Au}-\mathrm{C}_{16} \mathrm{TAB}-\mathrm{TC}_{8} \mathrm{AB}$ system. The electrolysis was carried out for 45 minutes with an applied current of $5 \mathrm{~mA}$ at a temperature of $42^{\circ} \mathrm{C}$ under constant ulterasonication. The centrifugation method was used at $6000 \mathrm{rpm}$ for $10 \mathrm{~min}$., and $18000 \mathrm{rpm}$ for $20 \mathrm{~min}$ at $30^{\circ} \mathrm{C}$ to separate the gold nanorods from the spherical nanoparticles. Depending on the ratio between hydrophilic and hydrophobic cationic surfactant, different nanorods of different aspect ratios were prepared. The spectroscopic characterization of the $\mathrm{Nds}$ solution was carried out by using of a Beckman DU 650 UVVisible spectrophotometer with matched quartz cells with pathlength $1 \mathrm{~cm}$. A cuvette holder was equipped with a
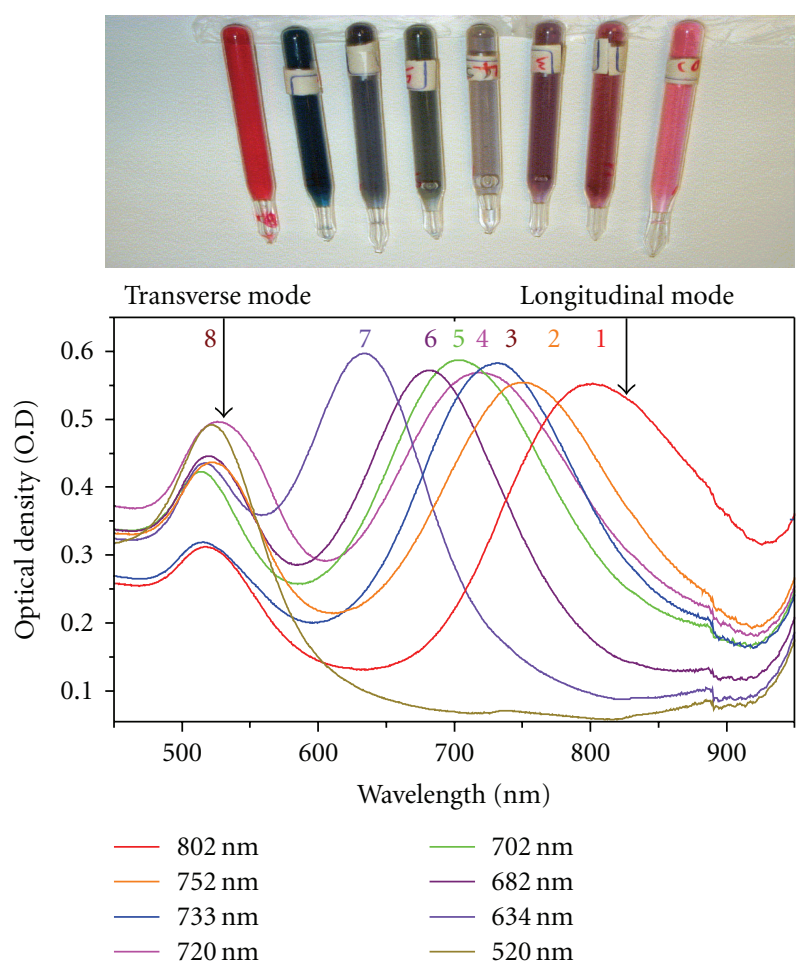

Figure 1: UV- vis absorption spectra of different gold nanorods in micellar solution.

Neslab RTE-100 thermostat to control the temperature of the sample under study. The sizes of the gold nanorods of different aspect ratios were determined from the position of the absorption maximum of transverse and longitudinal mode according to Link and El Sayed [12]. The changes in the absorption spectra of Nds solution were determined at different glycerol/deionized water ratios and at different temperatures. The glycerol used was $99.9 \%$ (Sigma) and the water was purified by ion exchange. The sample was placed in the heating cell holder, and the absorption spectrum of the sample was measured as soon as the sample solution become in equilibrium with the thermostat temperature.

\section{Results and Discussion}

The spherical gold nanospheres were prepared following a method introduced by Turkevich et al. [17]. Gold nanorods with different average aspect ratios were prepared as described above. Figure 1(a) shows the absorption spectra of eight different sizes of gold nanoparticles. It is known that the optical absorption of metallic nanoparticles is due to the surface plasmon (SP) resonance band(s).

The spherical gold nanoparticles have red color and showed only one plasmon absorption band at shorter wavelength, $\left(\lambda_{\max }=528 \mathrm{~nm}\right)$. On the other hand, the absorption spectra of $\mathrm{Au}$ nanorods are characterized by the intense longitudinal $\left(\mathrm{SP}_{L}\right)$ band at longer wavelength corresponding to the longitudinal resonance band and a much weak transverse resonance band at ca $\lambda_{\max }=528 \mathrm{~nm}$ [16]. The position of the longitudinal band undergoes red 
shift with increasing the gold nanorods aspect ratio. It is of interest to illustrate that the absorption spectra of the gold nanorods differ according to the aspect ratio as well as the amount of $\mathrm{Au}$ spheres present as impurity in the dispersions of gold nanorods. The average aspect ratios controlled by adjusting the concentration of surfactant molecules to cosurfactant molecules in the molar ratio $R=13-16$, because the the different sizes of gold nanorods produced depending on the different concentration ratios. The mean transverse diameter of a $\mathrm{Au}$ nanorods is typically equal to $\sim .10 \mathrm{~nm}$, while its mean longitudinal length is variable [14]. Figure 2 depicts the linear relationship between the aspect ratios $(R)$ and the wavelength of the absorption maxima of longitudinal plasmon resonance $\mathrm{SP}_{L}$ of the experimentally prepared gold. Such results reveal that the position of the $\left(\mathrm{SP}_{L}\right)$ band is strongly dependent on the aspect ratios of the Au nanorods, and the medium dielectric constant varies with the aspect ratio in nonlinear way. Chemically, this suggests that the structure of the capping micelle of the gold nanorods is size dependent [18]. The larger the aspect ratio is, the longer is the wavelength maximum of the $\mathrm{SP}_{L}$ band.

The studies of the effect of mixed solvents on the absorption spectra of gold nanorods were carried out for mixtures containing different ratios of water/glycerol (the percentage of water ranged from $25 \%$ to $75 \%$ ). Figures 3, 4, 5 show the absorption spectra and TEM of three different gold nanorods with aspect ratio of $4,3.5$, and 2.8 in binary solvents, with absorption maxima at 810,750 , and $680 \mathrm{~nm}$, respectively. The changes in the optical absorption spectra reflected the fact that the longitudinal plasmon resonance $\mathrm{SP}_{L}$ is strongly dependent on the dielectric constant of the binary solvents. The plasmon absorption is clearly visible and its maximum is red shifted with increasing the glycerol content. By correlating the dielectric constant of the pure components to the composition- dependent relation, the effect of binary solvent mixtures on the transition energy of absorption spectra of the nanorods could be calculated depending on the medium dielectric constant $(\varepsilon)$ as follow [19]:

$$
\operatorname{Ln} \varepsilon_{m}=X_{w} \operatorname{Ln} \varepsilon_{w}+X_{g} \operatorname{Ln} \varepsilon_{w}-0.3\left(X_{w} \cdot X_{g}\right),
$$

where $\varepsilon_{m}, \varepsilon_{w}, \varepsilon_{g}$ are the dielectric constants of the mixture, dielectric constants of water, and dielectric constants of glycerol, respectively. $X_{w}$ and $X_{g}$ are the mole fraction of water and glycerol and

$$
E_{T}^{(14)}=h c \bar{v} N A=2.859 \times 10^{-3} \bar{v} / \mathrm{cm}^{-1},
$$

where $E_{T}$ is the observed transition energy in kilocalories $/ \mathrm{mol}, \bar{v}$ is the wave number and NA is Avogadro's number. The effect of the medium dielectric constant of nanorods of different aspect ratio on the transition energy $\Delta \mathrm{E}$ of $\mathrm{SP}_{L}$ band is shown in Figure 6. A matual dramatic shift in the transition energy was observed on going from more dilelectric constant solvent $\left(\mathrm{H}_{2} \mathrm{O}\right)$ to lower dielectric constant solvent (glycerol). This illustrates that it depends on the medium dielectric constant of the complex which is intermediat between those of two solvents. The absorption maximum of $\mathrm{SP}_{L}$ band is

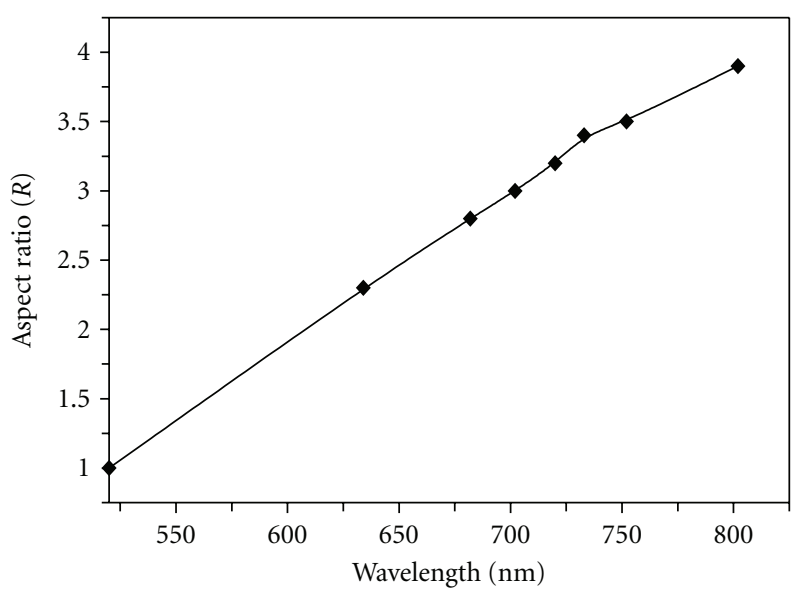

FIGURE 2: Show the linear relationship of the longitudinal band $\mathrm{SP}_{L}$ versus mean aspect ratio of the goldnanorods.

red shifted with increasing the glycerol/water ratio, while that of the $\mathrm{SP}_{t}$ transverse resonance remains constant at ca $\lambda_{\max }=528 \mathrm{~nm}$. The observed red shift for the solution of the different nanorods may be attributed to changes in the dielectric constant of the immediate environment surrounding a given particle. The optical density of $\mathrm{SP}_{L}$ band decreases slightly, while that of the $\mathrm{SP}_{t}$ transverse resonance band was found to increase at higher glycerol/water ratio. This might be due to some aggregation. It is expected that, when the dielectric constant of the medium increases the $\mathrm{SP}_{L}$ band shift to higher energies [20]. It is of interst to mention that, the bathochromic shift which accopmany the increasing of the glycerol ratio resulted from the increasing in the polydispersity of the glycerol in aqueous binary solvent mixtures. The micellar deformation rearranged around the small hydrophobic surface (glycerol) decreasing the overall dielectric constant. Sherbini et al. [21] studied the effect of the dielectric constant and the effect of hydrophobic surface on the c.m.c in binary glycerol/water ratios. The results obtained illustrated that the smaller in hydrophobic surface, and the larger in dielectric constant, the weakest the effect on the denaturation of c.m.c. The results also showed that, even at the small ratio of glycerol (5\%) the c.m.c was $4 \times 10^{-2} \mathrm{~mol} \mathrm{dm}^{-3}$ for sodium dodecyl sulphate (SDS), and for cetyltrimethyl ammonium bromide (CTAB) the c.m.c was $1.3 \times 10^{-2} \mathrm{~mol} \mathrm{dm}^{-3}$ in ethylene glycol [19]. This means that, when aqueous micellar solution are mixed with glycerol as a cosolvent, micellar fusion is expected to involve communication between the internal micellar phases. This might take place because the micelles interact preferentially with glycerol than with water. So, glycerol which is an analogous to water, can easily aggregate the micelles encapsulating the gold nanorods causing the longitudinal plasmon resonance $\left(\mathrm{SP}_{L}\right)$ to be red shifted. On the other hand, the study of the activation energy of the thermal instability of gold nanorods was carried out in aqueous micellar solution and in micellar solution of binary mixture at glycerol ratio of $75 \%$. The results obtained showed that the activation energy is found to be $12.9 \pm 1 \mathrm{k} \mathrm{cal} / \mathrm{mol}$ in the binary mixture in 


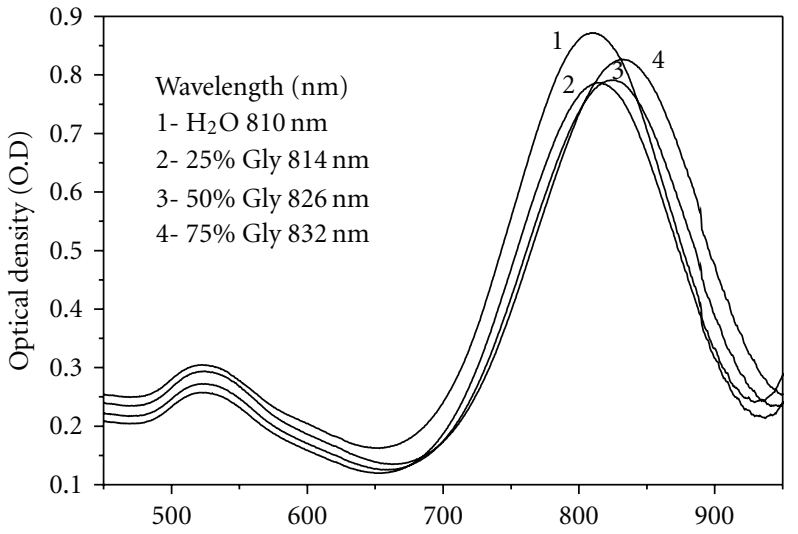

(a)

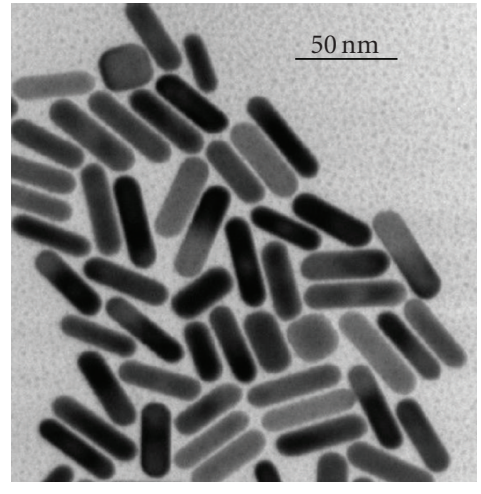

(b)

Figure 3: (a) Absorption spectra of gold nanorods with aspect ratio $L / d=4$ in 1 -water, $2 \%-25 \%$, 3\%-50\%, and 4\%-75\% of aqueous glycerol, (b) (TEM) in aqueous micellar solution.

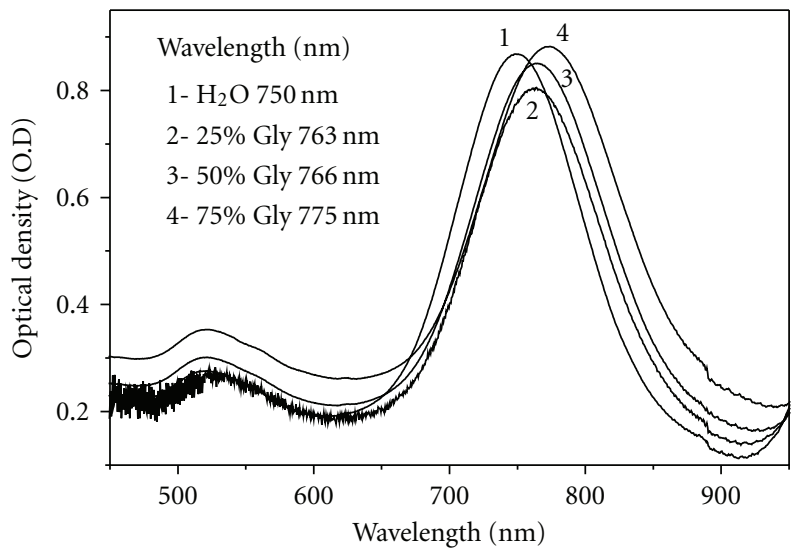

(a)

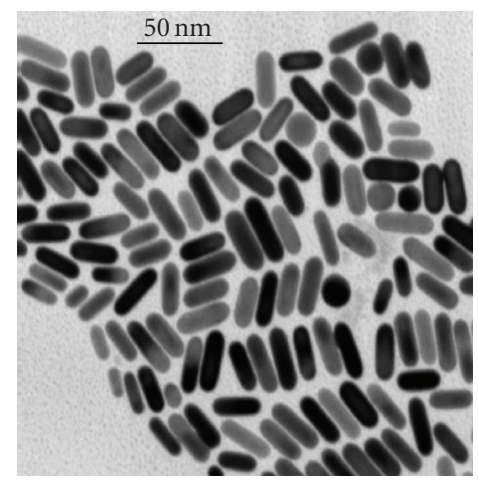

(b)

Figure 4: (a) Absorption spectra of gold nanorods with aspect ratio $L / d=3.5$, (b) TEM 1-water, $2 \%-25 \%$, 3\%-50\%, and 4\%-75\% of aqueous glycerol, (b) (TEM) in aqueous micellar solution.

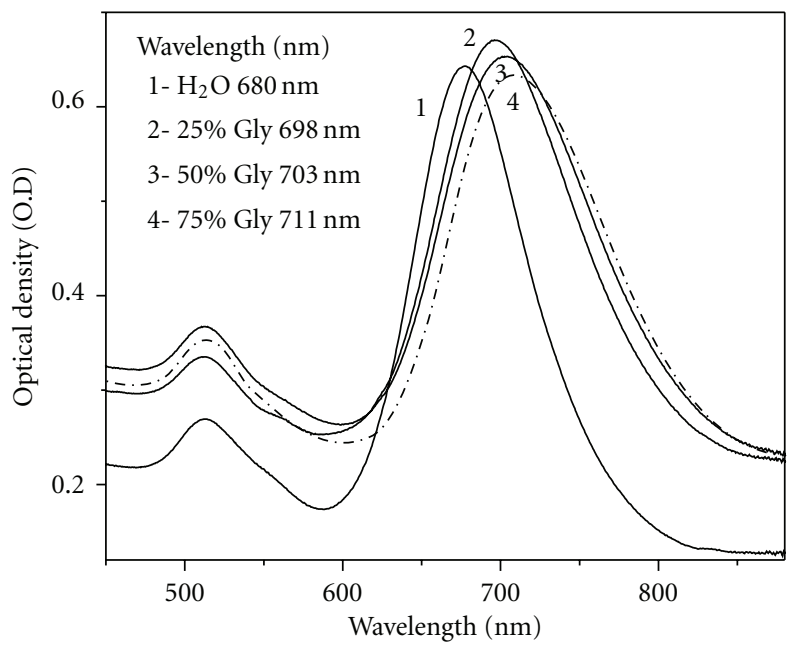

(a)

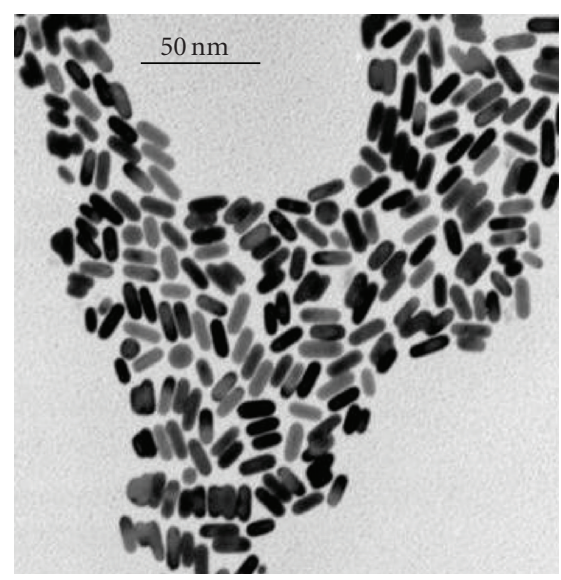

(b)

Figure 5: (a) Absorption spectra of gold nanorods with aspect ratio $L / d=2$. 1-water, $2 \%-25 \%, 3 \%-50 \%$, and 4\%-75\% of aqueous glycerol, (b) (TEM) in aqueous micellar solution, (b) (TEM) in micellar solution. 


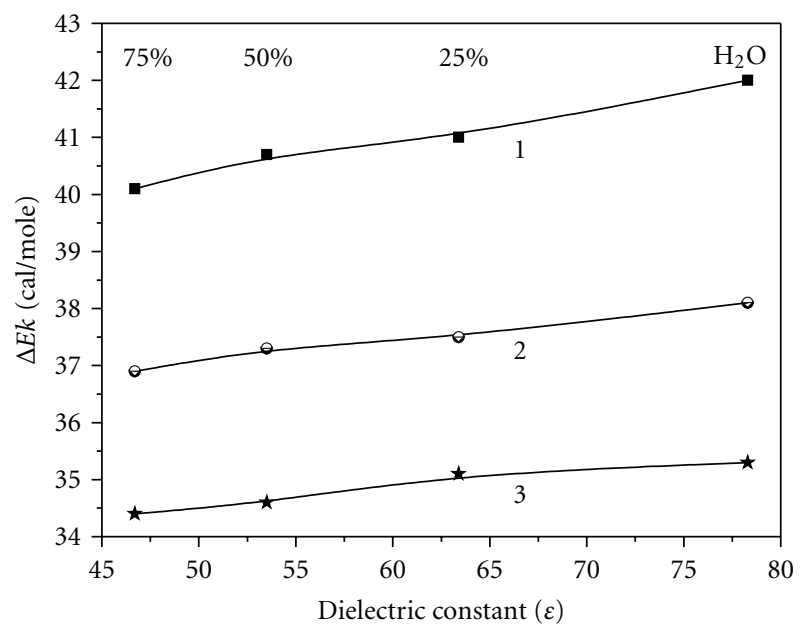

Figure 6: The relationship of the dielectric constant with the transition energy of $1-4,2-3.5$, and $3-2.8$ aspect ratio of of the goldnanorods in aqueous Glycerol (25\%-75\%).

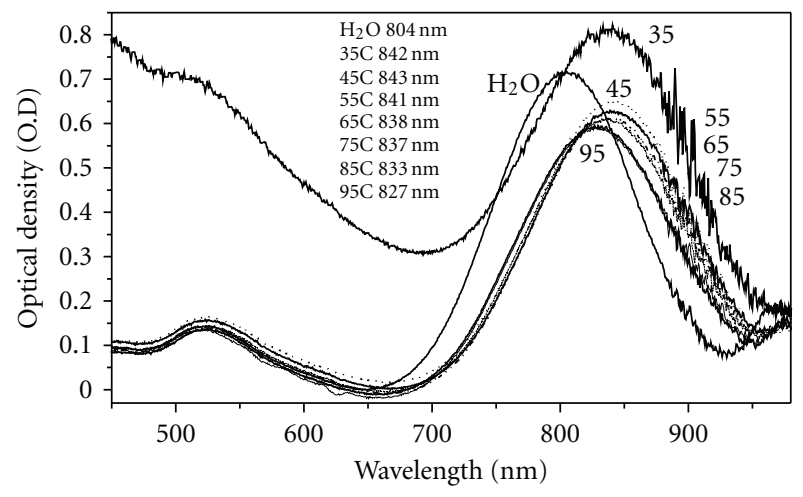

FIGURE 7: The optical absorption spectra of $L / d=4$ gold nanorod in Glycerol/Water Ratio of $75 \%(\mathrm{v} / \mathrm{v})$ at different temperatures $(25 \%-75 \%)$.

comparison with the value obtained by Mahmoud et al. [22] which is $21.0 \pm 1 \mathrm{k} \mathrm{cal} / \mathrm{mol}$ in micellar solution. The decrease in the activation energy of the same aspect ratio of gold nanorods can be explained on the basis of preferential solvation by glycerol than by water causing decreases in the overall medium dielectric constants. The effect of heating on the gold nanorods solutions of average aspect ratio 4 and 3.5 with $\mathrm{SP}_{L}$ band maxima at $810,752 \mathrm{~nm}$, respectively, was studied between room temperature and $95^{\circ} \mathrm{C}$. By raising the temperature, the absorption maximum of $\mathrm{SP}_{L}$ band exhibits a measurable change in the optical density. This is due to the change of the colloidal solution from turbid to transparent solution. With increasing the temperatures gradually, the spectrum starts to blue shift. A maximum value of $\sim 14 \mathrm{~nm}$ and $17 \mathrm{~nm}$ is observed for the two nanoparticles of average aspect ratios 4 and 3.5, respectively, (see Figures 7). This suggests that, by increasing the temperature, the average aspect ratio of the gold nanorods and their distribution decreases, (in the solution). The observed blue shift of the absorption maximum accompanies the temperature increases may be resulted from the differential thermal instability of rods or the capping micelle. The heat could reshape the individual nanorods inside the micelles, or the long micelles could dissolve at higher temperature increases. The later leads to percipitation of the long gold nanoparticles out of the solution being studied [22]. The previous work has shown that it is the higher solubility of the longer miscelles at higher temperature that leads to the observed blue shift of the rods absorption. The longer micelles are not as stable as the shorter ones. This leads to a decrease in the average aspect ratio, with the observed blue shift of the $\mathrm{SP}_{L}$ band maximum [22], and there are no significant changes in the width of the nanorods with increasing the temperatures. Therefore, it is concluded that the shift of the $\mathrm{SP}_{L}$ absorption maximum and the corresponding reduction in the average aspect ratio are caused by decrease in the lengths of the nanoparticles present in solution. By comparing the results obtained by heating the rods of different aspect ratios, we found that the medium dielectric constant is also temperature dependent. This attributed to the thermal annealing of the structure of the micelles around the nanorods. Again, Figure 7 shows a very slight increase in the optical density of the absorption maximum of $\mathrm{SP}_{L}$ and $\mathrm{SP}_{t}$ due to the increase in the light scattering of the turbid solution and also the slight $(5 \mathrm{~nm})$ red shift of the absorption maximum of the $\mathrm{SP}_{L}$ band as the solution cools down to room temperature. Such reversible slight red shift observed may be resulted from the decrease in the dielectric constant of the surrounding medium with decreasing the temperature. Otherwise, the gold nanorods will melt and they are not reshaped to the longer size. The absorption spectra of the thermal instability of the nanorods was studied at $130^{\circ} \mathrm{C}$. the results showed that the surface plasmon absorption spectra of the transverse and longitudinal bands completely disappear after $30 \mathrm{~min}$. This suggests that, the micelles encapsulating the gold nanoparticles are completely destroyed, and an observed black precipitate of aggregated gold powder is formed. This means that, the decomposition temperature of the most stable micelles used in this work is at $\sim 130^{\circ} \mathrm{C}$, while in case of Mahmoud et al. [22] was in the range of $155-160^{\circ} \mathrm{C}$.

\section{Conclusion}

In the presence of glycerol water cosolvent, the longitudinal surface plasmon resonance $\left(\mathrm{SP}_{L}\right)$ is strongly dependent on the dielectric constant of the mixtures ratio and temperatures. The binary solvents at any mixtures used in the study can easily dissociate or associate the micelles encapsulated by the gold nanoparticles.

In case of the temperature change, the longitudinal surface plasmon resonance $\left(\mathrm{SP}_{L}\right)$ varies with both of temperature changes and the relative instability of the nanorods' capping micelles with increasing the temperature.

\section{Acknowledgments}

The author gratefully acknowledges the help of Professor M. A. El-Sayed the director of laser dynamics lab (LDL) at 
the school of chemistry and biochemistry, Georgia Institute of Technology for his many helpful discussions during carrying out this work in his Laboratory. The author also acknowledges his excellent students especially Ms. Mona Mohamed who helped in carrying out this work in his lab. Also, special thanks to the Egyptian Government for the fellowship.

\section{References}

[1] V. Sharma, K. Park, and M. Srinivasarao, "Colloidal dispersion of gold nanorods: historical background, optical properties, seed-mediated synthesis, shape separation and self-assembly," Materials Science and Engineering R, vol. 65, no. 1-3, pp. 1-38, 2009.

[2] M.-C. Daniel and D. Astruc, "Gold nanoparticles: assembly, supramolecular chemistry, quantum-size-related properties, and applications toward biology, catalysis, and nanotechnology," Chemical Reviews, vol. 104, no. 1, pp. 293-346, 2004.

[3] J. Pérez-Juste, I. Pastoriza-Santos, L. M. Liz-Marzán, and P. Mulvaney, "Gold nanorods: synthesis, characterization and applications," Coordination Chemistry Reviews, vol. 249, no. 17-18, pp. 1870-1901, 2005.

[4] A. V. Alekseeva, V. A. Bogatyrev, B. N. Khlebtsov, A. G. Mel'nikov, L. A. Dykman, and N. G. Khlebtsov, "Gold nanorods: synthesis and optical properties," Colloid Journal, vol. 68 , no. 6, pp. 661-678, 2006.

[5] L. A. Dykman and V. A. Bogatyrev, "Gold nanoparticles: synthesis, functionalization, the use in biochemistry and immunochemistry," Uspekhi Khimii, vol. 76, no. 2, pp. 199213, 2007.

[6] C. Burda, X. Chen, R. Narayanan, and M. A. El-Sayed, "Chemistry and properties of nanocrystals of different shapes," Chemical Reviews, vol. 105, no. 4, pp. 1025-1102, 2005.

[7] M. A. El-Sayed, "Small is different: shape-, size-, and composition-dependent properties of some colloidal semiconductor nanocrystals," Accounts of Chemical Research, vol. 37, no. 5, pp. 326-333, 2004.

[8] A. P. Alivisatos, "Perspectives on the physical chemistry of semiconductor nanocrystals," Journal of Physical Chemistry, vol. 100, no. 31, pp. 13226-13239, 1996.

[9] M. Kerker, The Scattering of Light and Other Electromagnetic Radiation, Academic Press, New York, NY, USA, 1969.

[10] C. F. Bohren and D. R. Huffman, Absorption and Scattering of Light by Small Particles, John Wiley, New York, NY, USA, 1983.

[11] G. Mie, "Beiträge zur optik trüber medien, speziell kolloidale metallösungen," Annalen der Physik, vol. 330, no. 3, pp. 377445, 1908.

[12] S. Link and M. A. El-Sayed, "Size and temperature dependence of the plasmon absorption of colloidal gold nanoparticles," Journal of Physical Chemistry B, vol. 103, no. 21, pp. 42124217, 1999.

[13] M. S. Bakshi, "Micelle formation by anionic and cationic surfactants in binary aqueous solvents," Journal of the Chemical Society, Faraday Transactions, vol. 89, no. 24, pp. 4323-4326, 1993.

[14] M. Roses, C. Rafols, J. Ortega, and E. Bosch, "Solute-solvent and solvent-solvent interactions in binary solvent mixtures. Part 1. A comparison of several preferential solvation models for describing $E_{\mathrm{T}}(30)$ polarity of bipolar hydrogen bond acceptor-cosolvent mixtures," Journal of Chemical Society Perkin Transactions, vol. 2, no. 8, pp. 1607-1615, 1995.
[15] S.-S. Chang, C.-W. Shih, C.-D. Chen, W.-C. Lai, and C. R. Chris Wang, "The shape transition of gold nanorods," Langmuir, vol. 15, no. 3, pp. 701-709, 1999.

[16] Y.-Y. Yu, S.-S. Chang, C.-L. Lee, and C. R. Chris Wang, "Gold nanorods: electrochemical synthesis and optical properties," Journal of Physical Chemistry B, vol. 101, no. 34, pp. 66616664, 1997.

[17] J. Turkevich, P. C. Stevenson, and J. Hillier, "A study of the nucleation and growth processes in the synthesis of colloidal gold," Discussions of the Faraday Society, vol. 11, pp. 55-75, 1951.

[18] S. Link, M. B. Mohamed, and M. A. El-sayed, "Simulation of the optical absorption spectra of gold nanorods as a function of their aspect ratio and the effect of the medium dielectric constant," Journal of Physical Chemistry B, vol. 103, no. 16, pp. 3073-3077, 1999.

[19] R. Nagarajan and C.-C. Wang, "Theory of surfactant aggregation in water/ethylene glycol mixed solvents," Langmuir, vol. 16, no. 12, pp. 5242-5251, 2000.

[20] J. R. Heath, C. M. Knobler, and D. V. Leff, "Pressure/temperature phase diagrams and superlattices of organically functionalized metal nanocrystal monolayers: the influence of particle size, size distribution, and surface passivant," Journal of Physical Chemistry B, vol. 101, no. 2, pp. 189-197, 1997.

[21] E.-S. A. M. Al-Sherbini, M. H. Abdel-Kader, and R. Y. Hamzah, "Effect of binary solvents on the critical micelles concentration by using 1-methyl-4-[4'-aminostyryl]pyridinium iodide," Colloids and Surfaces A: Physicochemical and Engineering Aspects, vol. 194, no. 1-3, pp. 133-142, 2001.

[22] M. B. Mohamoud, K. Z. Ismail, S. Link, and M. A. El-sayed, "Thermal reshaping of gold nanorods in micelles," Journal of Physical Chemistry B, vol. 102, no. 47, pp. 9370-9374, 1998. 

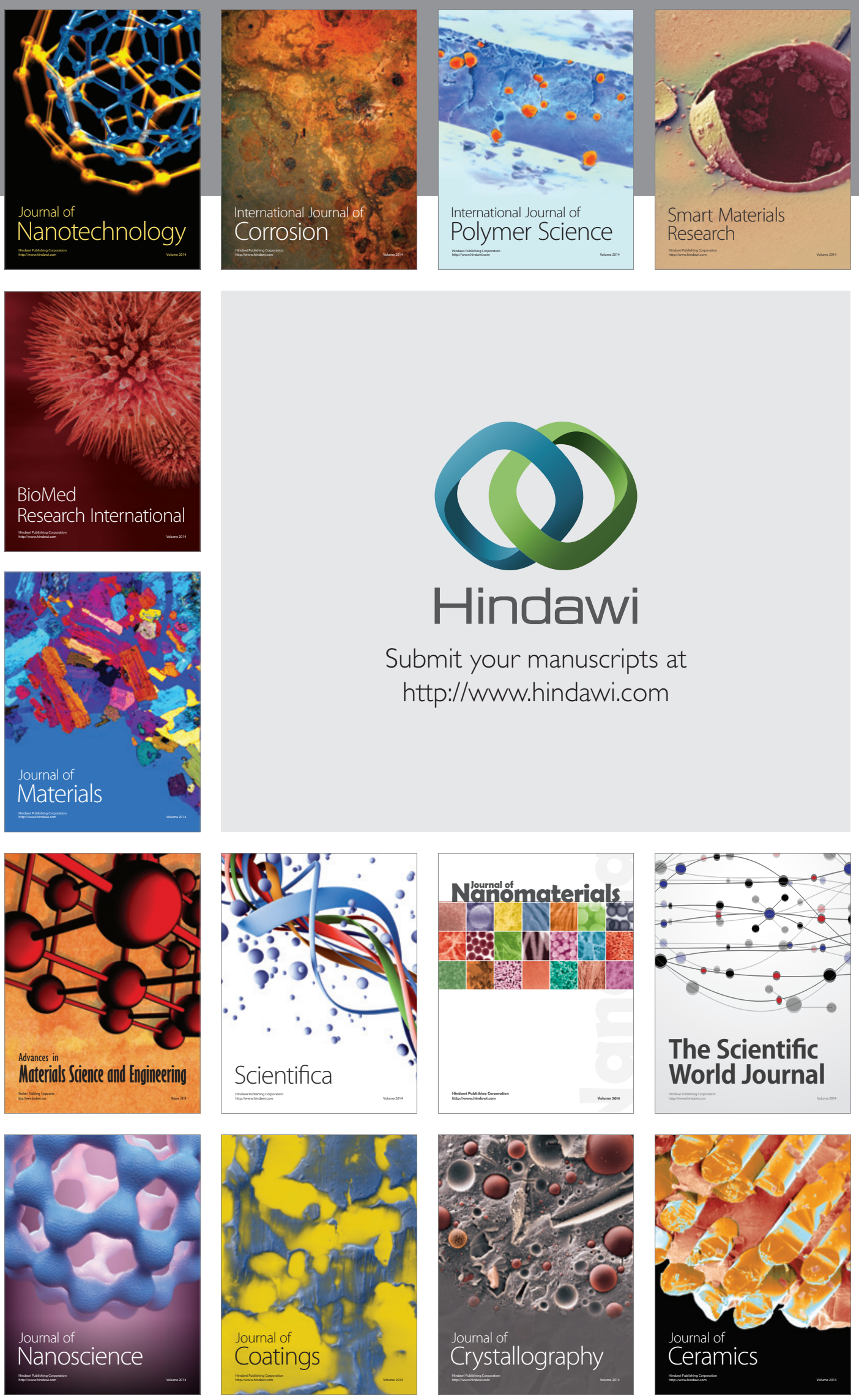

The Scientific World Journal

Submit your manuscripts at

http://www.hindawi.com

\section{World Journal}

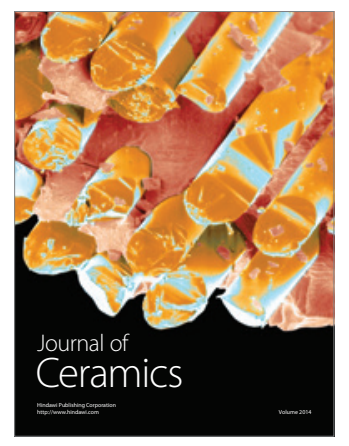

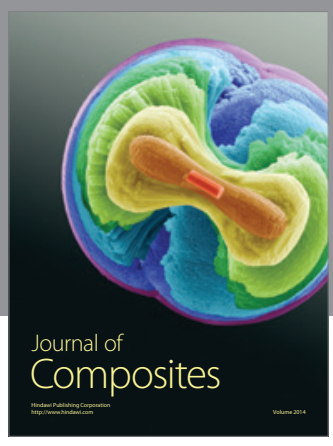
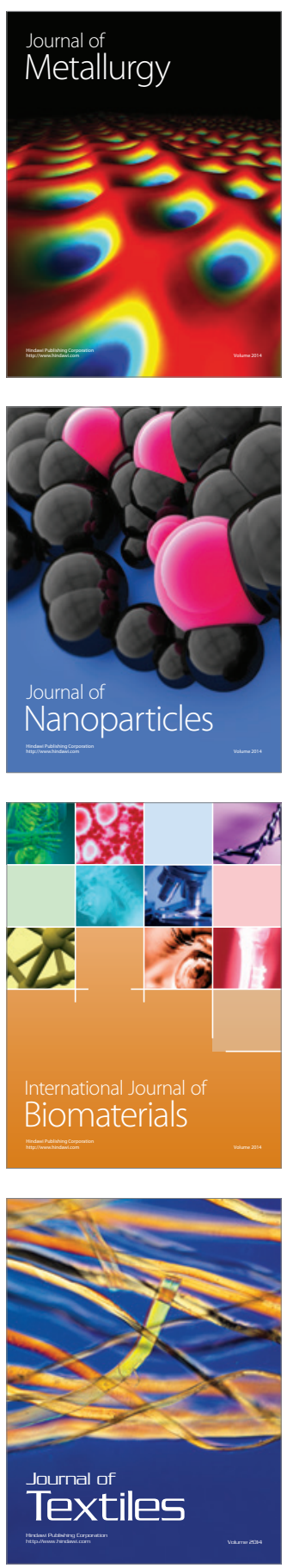